\title{
ATENÇÃO À SAÚDE DE PESSOAS COM DIABETES MELLITUS E HIPERTENSÃO ARTERIAL EM UNIDADE LOCAL DE SAÚDE: RELATO DE EXPERIÊNCIA
}

\author{
Gabriela Ricken Warmling ${ }^{1}$ \\ Juliana Cristina Lessmann \\ Betina Hörner Schlindwein Meirelles ${ }^{3}$ \\ Denise Guerreiro V. da Silva ${ }^{4}$
}

\section{RESUMO}

Trata-se de relato de experiência dos participantes do projeto de extensão "Atenção à saúde de pessoas com diabetes mellitus e hipertensão arterial na Unidade Local de Saúde da Lagoa da Conceição, Florianópolis, SC". Foram realizadas consultas de enfermagem e visitas domiciliares ao público-alvo, visando proporcionar um viver mais saudável, contribuir com o desenvolvimento de atividades de enfermagem integrais e de qualidade, além de fortalecer o vínculo destas pessoas com o serviço local de saúde.

Palavras-chave: Enfermagem. Doença crônica. Visita domiciliar.

\footnotetext{
${ }^{1}$ Acadêmica do Curso de Enfermagem da Universidade Federal de Santa Catarina. gabrielarf13@yahoo.com.br

${ }^{2}$ Mestre em Enfermagem pela Universidade Federal de Santa Catarina - UFSC. Professora Substituta do Departamento de Enfermagem da UFSC. julianalessmann@gmail.com

${ }^{3}$ Doutorado em enfermagem pela universidade Federal de Santa Catarina - UFSC. Professora do Departamento de Enfermagem da Universidade Federal de Santa Catarina. Coordenadora do projeto. betinam@ccs.ufsc.br

${ }^{4}$ Pós-Doutorado pela Faculty of Nursing University of Alberta. Professora do Departamento de Enfermagem da Universidade Federal de Santa Catarina. Coordenadora do NUCRON. denise@nfr.ufsc.br
} 


\title{
HEALTH CARE OF PEOPLE WITH DIABETES MELLITUS AND HYPERTENSION IN THE LOCAL HEALTH UNIT: REPORT OF EXPERIENCE
}

\begin{abstract}
This is an experience report of participating in the extension project entitled "Health care of people with diabetes mellitus and hypertension in the Local Health Unit of Lagoa da Conceição, Florianópolis, SC". Were performed nursing consultations and home visits to the target audience, aiming to provide a healthier lifestyle, contribute to the development of activities with quality and integral nursing, in addition to strengthen the link between these people with the local health service.
\end{abstract}

Keywords: Nursing. Chronic disease. Home visit.

\section{INTRODUÇÃO}

Conviver com doenças crônico-degenerativas demanda adaptações ao estilo de vida que envolve controle medicamentoso, alimentar, prática de atividade física e ações que comumente não são executadas antes da descoberta da doença. Assim, estabelecer hábitos de vida saudáveis para o gerenciamento adequado da doença engloba modificações individuais bem como o suporte social e de saúde (SILVA, 2001).

$\mathrm{O}$ acompanhamento à saúde de pessoas com doenças crônicas promove o aumento da qualidade de vida, a redução do estabelecimento de co-morbidades e de agravos secundários como problemas cardíacos, perda da função renal, diminuição da função visual, acidente vascular encefálico e amputação de membros inferiores. Neste sentido, discutir o cuidado em enfermagem para as pessoas com hipertensão arterial e diabetes mellitus assume relevância, considerando as inúmeras complicações que podem ocorrer em decorrência dessas doenças.

A hipertensão arterial sistêmica (HAS) é caracterizada como "pressão arterial sistólica maior ou igual a $140 \mathrm{mmHg}$ e uma pressão arterial diastólica maior ou igual a $90 \mathrm{mmHg}$, em indivíduos que não estão fazendo uso de medicação anti-hipertensiva” (BRASIL, 2006a, p. 14). Esta doença acomete um número significativo de pessoas e gera muitas complicações ao 
organismo, o que reduz a qualidade de vida a médio e longo prazo, além de caracterizar-se num risco o sistema cardiovascular e renal (BRASIL, 2002).

O diabetes mellitus (DM) é uma doença crônica bastante prevalente na população, sendo reconhecido como "um grupo de doenças metabólicas caracterizadas por hiperglicemia e associadas a complicações, disfunções e insuficiência de vários órgãos, especialmente olhos, rins, nervos, cérebro, coração e vasos sangüíneos" (BRASIL, 2006b, p. 09). Esta doença pode ser decorrente da diminuição na produção de insulina pelo pâncreas ou no aumento de resistência a ação da insulina nas células alvo (CIPRIANO et al., 2006).

O diagnóstico do DM é realizado por meio da história clínica, envolvendo algumas sintomatologias clássicas como poliúria, polidipsia, polifagia, perda involuntária de peso, dentre outros, além de exames laboratoriais (BRASIL, 2002). Cabe ressaltar que esta doença é classificada em três tipos, que ocorrem mais frequentemente, sendo o tipo 1 ou diabetes juvenil; o tipo 2 ou diabetes do adulto; e o diabetes gestacional, detectado durante o pré-natal, cuja etiologia não está totalmente elucidada (BRASIL, 2002).

A proposta de desenvolver uma atividade de extensão com pessoas com diabetes mellitus (DM) e hipertensão arterial sistêmica (HAS) está alicerçada na experiência e conhecimentos que o Núcleo de Estudos e Assistência a pessoas com Doenças Crônicas (NUCRON) vem adquirindo em suas atividades de pesquisa, ensino e assistência realizadas nos últimos 20 anos.

O NUCRON foi criado em 1987 e, desde seu início, focalizou suas investigações em pessoas com doenças crônicas, especialmente aquelas com DM, HAS, doença pulmonar obstrutiva crônica, asma, estomias, doença arterial coronariana e insuficiência renal crônica. O NUCRON é um grupo de pesquisa vinculado ao Departamento de Enfermagem e ao Programa de Pós-Graduação em Enfermagem da Universidade Federal de Santa Catarina (UFSC). É um grupo consolidado que está registrado junto ao Conselho Nacional de Desenvolvimento Científico e Tecnológico (CNPq).

O NUCRON tem produzido conhecimentos a partir de pesquisas que realiza, vinculando-as ao ensino e à prática assistencial em saúde. Destacamos as temáticas abordadas:

a) Enfrentamento e saúde: sistematização do processo de viver em uma condição crônica de saúde; diferentes tendências na construção da doença crônica; construção e validação de instrumentos de pesquisa e de assistência; 
conceitualização do processo de enfrentamento de uma condição crônica de saúde.

b) Qualidade de vida: a formação de grupos de convivência; métodos e técnicas para o desenvolvimento de educação em saúde em grupos; elementos que integram o que as pessoas em condição crônica consideram como qualidade de vida.

c) Promoção da saúde: concepção de saúde e cronicidade; desenvolvimento de uma estrutura teórica e de estratégias para tornar operacional a promoção da saúde; processo de intercomunicação intra e inter grupos de profissionais da saúde e de pessoas que vivem com uma condição crônica.

d) Itinerário terapêutico: Sistema de Cuidado à Saúde; Subsistema Profissional, Popular e Familiar; Itinerário Terapêutico de pessoas com doenças crônicas (SILVA; MEIRELLES, 2008). Estas temáticas constituem o referencial utilizado e as atividades propostas no grupo.

Neste sentido, foi proposto pelos integrantes do NUCRON o desenvolvimento desta atividade, cujo foco está no cuidado às pessoas com HAS e DM. Foram desenvolvidas ações conjuntas envolvendo a acadêmica bolsista; membros do grupo de pesquisa NUCRON, incluindo professores e alunos da graduação e da pós-graduação em Enfermagem; agentes comunitários de saúde; médicos e enfermeiros da Unidade Local de Saúde (ULS) envolvida, visando a integralidade do serviço de saúde às pessoas portadoras de HAS e DM.

Assim, apresentamos o relato das atividades de extensão desenvolvidas na "Atenção à saúde de pessoas com diabetes mellitus e hipertensão arterial na Unidade Local de Saúde da Lagoa da Conceição”, que articulou atividades de ensino, pesquisa e extensão. O projeto teve como objetivo desenvolver atenção à saúde de pessoas com DM e HAS na Unidade Local de Saúde da Lagoa da Conceição (ULS/LC) - Florianópolis/SC.

\section{MATERIAL E MÉTODOS}

Trata-se de um relato de experiência vivenciada na realização de projeto de extensão desenvolvido junto à ULS da Lagoa da Conceição, na cidade de Florianópolis/Santa Catarina, no período de março a dezembro de 2008. Esta ULS conta com duas equipes de Estratégia Saúde da Família (ESF), e, segundo dados obtidos da Prefeitura Municipal de Florianópolis, 
em 2008, residem, em sua área de abrangência, 8.185 pessoas, das quais 608 têm de 50 a 59 anos de idade e 555 possuem mais de 60 anos (PMF, 2008).

A prevalência de pessoas portadoras de diabetes e hipertensão arterial aumenta com o avanço da idade, sendo fundamental o desenvolvimento de atividades de prevenção e promoção da saúde com a população, bem como ofertar atendimento qualificado a esta clientela (BRASIL, 2002). Segundo o Ministério da Saúde (2008), uma ULS destina-se à realização de atendimento de atenção básica e integral a uma população de forma programada ou não nas especialidades básicas, podendo oferecer assistência odontológica e de outros profissionais de nível superior. Assim, a ULS da Lagoa da Conceição foi selecionada diante dos seus objetivos e por ser local no qual o NUCRON já desenvolvia atividades de pesquisa e ensino.

A metodologia de ação desta atividade de extensão visou o fortalecimento dos laços entre pesquisadores, alunos e os profissionais de saúde; o reconhecimento das necessidades da ULS e da comunidade; a elaboração de protocolo de consulta e visita domiciliar; a implantação de agenda para atendimento da comunidade; a realização de consultas e visitas domiciliares de enfermagem; a realização de ações educativas aos agentes comunitários de saúde.

A atuação junto à ULS iniciou com a explanação do projeto de extensão às equipes médicas e de enfermagem, bem como aos agentes comunitários de saúde. Neste momento foi possível sensibilizá-los quanto à importância da implantação do projeto e da participação destes para termos acesso às pessoas com DM e HAS residentes na comunidade.

Os integrantes do NUCRON iniciaram então, junto com os enfermeiros da ULS, o agendamento de consultas para as quartas-feiras no período vespertino. Foi elaborada uma escala com o agendamento das participações semanais dos membros do NUCRON.

A partir da dificuldade inicial de realização de consultas de enfermagem, buscamos identificar as necessidades das pessoas com DM e HAS através de realização de visitas domiciliares. O grupo de convivência, que já vinha sendo realizado com a participação de um dos integrantes do NUCRON, com encontros mensais, na sede da Sociedade Amigos da Lagoa (SAL), foi mantido com o objetivo de ampliar a participação das pessoas no grupo através de orientação e encaminhamento.

Os protocolos de consulta e de visita domiciliar de enfermagem foram elaborados após rigorosa busca em bases de dados e discussões, nos meses de março a junho de 2008. Esta 
construção do modelo de consulta e de visita domiciliar de enfermagem foi realizada a partir das necessidades da ULS e do conhecimento desenvolvido pelo NUCRON no cuidado a pessoas com doenças crônicas, com a participação dos profissionais da ULS e em discussões nas reuniões do grupo de pesquisa.

A atuação da bolsista de graduação sempre foi acompanhada, com supervisão direta de integrantes do NUCRON e enfermeiros da ULS. As atividades de extensão iniciaram após a autorização para a realização conferida pela Secretaria Municipal de Saúde e da coordenação da ULS.

Participaram da atividade de extensão cerca de 14 membros NUCRON, sendo sete enfermeiras, um professor de educação física, um fisioterapeuta e quatro estudantes de graduação, que contribuíram diretamente para o desenvolvimento do projeto, seja nas discussões teóricas, seja na operacionalização do projeto.

\section{RESULTADOS E ANÁLISE}

A realização da atividade de extensão trouxe resultados efetivos e avanços no desenvolvimento do cuidado a pessoas com doenças crônicas, como discutiremos a seguir.

Observamos a melhoria da comunicação e vínculos entre os membros da equipe da ULS e integrantes do NUCRON, fortalecendo a relação entre Universidade Federal de Santa Catarina e ULS, que serve de campo de pesquisa, ensino e extensão.

A possibilidade da participação de alunos de graduação, mais especificamente de enfermagem nas atividades desenvolvidas, como a consulta e visitas domiciliares, favoreceu a aprendizagem e desenvolvimento de habilidades práticas no cuidado a pessoas com DM e HAS, que muitas vezes não é alcançada nas atividades curriculares.

O grupo de convivência de pessoas com HAS e DM foi desenvolvido com a participação de integrantes do NUCRON e da ULS, nas últimas segundas-feiras de cada mês, no período da tarde. Foram desenvolvidas novas possibilidades de orientação, integração e interação entre pessoas com doença crônica e o grupo técnico. Também houve participação nas atividades lúdico-educativas do grupo Viva a Vida, composto por pessoas idosas com DM e HAS. Na perspectiva do grupo de convivência, foram desenvolvidas atividades de educação e lazer entre pessoas com doenças crônicas e profissionais. Os grupos de convivência, segundo Francioni e Silva (2007), proporcionam a continuidade da atenção em saúde, a 
integração deste indivíduo na comunidade, o fortalecimento de relações afetivas e de suporte social.

Dessa forma, a realização das atividades foi integrada com as ações da equipe da ULS, que indicaram as pessoas que mais necessitavam de assistência em saúde, sendo realizados contatos telefônicos e pessoais para o agendamento de consultas de enfermagem na ULS.

As atividades ficaram sob a responsabilidade de uma enfermeira e da acadêmica de enfermagem, integrantes do NUCRON, sendo que para tal foram utilizados roteiros de consulta e de visita domiciliar de enfermagem, elaborados previamente, que continham orientações para a coleta de dados relacionados à identificação da pessoa, avaliação de hábitos de saúde, avaliação física geral e específica para a identificação precoce de agravos decorrentes do DM e HAS. O roteiro utilizado mostrou-se adequado à proposta, permitindo realizar as atividades de maneira sistemática e consistente.

A atuação junto à comunidade foi através do agendamento de consultas semanais, nas quartas-feiras, no período vespertino, sendo elaborada uma escala de enfermeiras do NUCRON para o atendimento à demanda. Transcorridos dois meses e meio, percebeu-se que havia resistência das pessoas em frequentar as consultas de enfermagem previamente agendadas, fato observado pelo elevado índice de ausências, o que exigiu novas estratégias para sensibilização do público-alvo. Esse fato foi um desafio a ser superado, o que ampliou o interesse na continuidade de ações que enfoquem esta perspectiva.

Diante da baixa adesão das pessoas às consultas, optou-se pela realização de visitas domiciliares previamente agendadas pela acadêmica bolsista e pelos agentes comunitários de saúde. Para tal, foi considerada a disponibilidade das pessoas, a possibilidade de acompanhamento do agente comunitário de saúde e a participação dos enfermeiros da ULS e NUCRON nas atividades.

Foram realizadas 62 visitas domiciliarias a pessoas com idades que variaram de 42 a 88 anos, de ambos os sexos, no período de julho a dezembro de 2008 , com o objetivo de promover a saúde e a prevenção de complicações decorrentes destas doenças crônicas. No momento das visitas, estavam sempre presentes uma enfermeira da ULS ou do NUCRON, bem como um agente comunitário de saúde e a acadêmica bolsista.

No decorrer da visitas eram verificadas a glicemia capilar e a pressão arterial; orientávamos quanto às questões alimentares, aos exercícios físicos e formas de viver bem com uma doença crônica; o enfrentamento de obstáculos para adesão ao tratamento e 
realização de cuidados. As pessoas visitadas detinham as mais diversas dificuldades em seguir o tratamento, como dependência de outras pessoas para tomar medicamentos, dificuldade em modificar o estilo de vida em função da doença, ou alegavam falta de tempo para cuidarem de si. Sempre que possível, sanávamos as suas dúvidas, bem como realizávamos pedidos para marcação de consulta com profissionais da ULS.

Com estas atividades, percebemos quão importante é o papel da equipe de saúde e dos agentes comunitários para a plena realização das visitas domiciliares, pois estes facilitam o acesso, a formação de vínculo e o estabelecimento de uma abordagem mais completa do processo saúde-doença das pessoas com DM e HAS.

Apesar de não termos efetuado uma avaliação sistemática da efetividade da prática assistencial desenvolvida, acreditamos que assistência realizada contribuiu na promoção da saúde das pessoas atendidas e também mobilizou os profissionais de saúde da ULS para a realização de consultas de enfermagem e visitas domiciliárias, utilizando o referencial teóricometodológico proposto pelo NUCRON.

Desta forma, esta oportunidade permitiu ampliar a percepção e compreender melhor o processo de viver com doença crônica, oportunizando o enriquecimento de conteúdos e o fortalecimento significativo das bases teórico-práticas acerca destas doenças, que são de grande relevância epidemiológica no atual quadro de saúde brasileiro (BRASIL, 2002). Ressalta-se também a contribuição significativa no reconhecimento do papel do enfermeiro na prevenção e controle de doenças crônicas, aliado ao conhecimento acadêmico, à prática profissional e ao relacionamento interpessoal.

\section{CONSIDERAÇÕES FINAIS}

As visitas domiciliárias possibilitaram a visualização dos diversos contextos nos quais vivem as pessoas com DM e HAS, suas dificuldades e fragilidades. Foi possível identificar a importância do profissional ao desenvolver a educação visando à promoção da saúde; o exercício do trabalho em equipe; o desenvolvimento das habilidades práticas no cuidado às pessoas com doenças crônicas; o aprofundamento do conhecimento teórico-prático acerca de DM e HAS e a rica experiência adquirida em realizar assistência com empatia, respeito, responsabilidade e humanização, em prol de um viver mais saudável.

O desenvolvimento desta atividade de extensão respondeu as expectativas do NUCRON em retornar à comunidade parte do conhecimento construído por meio das ATENÇÃO À SAÚDE DE PESSOAS COM DIABETES MELLITUS E HIPERTENSÃo ARTERIAL EM UNIDADE LOCAL DE 153 SAÚDE: RELATO DE EXPERIÊNCIA

Warmling, Lessmann, Meirelles, Silva. 
pesquisas e estudos desenvolvidos. Possibilitou a promoção de vivências com a realidade de pessoas em condição crônica na ULS da Lagoa da Conceição, que também já serve de campo para atividades práticas do ensino curricular do curso de Graduação em Enfermagem da UFSC.

Em face da importância de atenção à saúde de pessoas com DM e HAS e da dificuldade para a implantação de consultas de enfermagem para esta população devido à baixa aderência dos usuários, recomenda-se a realização de visitas domiciliares de conscientização acerca do serviço, das necessidades de controle das doenças e da disponibilidade dos profissionais junto às unidades de saúde. Assim, recomenda-se a realização de projetos com esta perspectiva para que tais atividades possam ser executadas, fortalecendo a relação da ULS com a comunidade e com a Universidade.

\section{REFERÊNCIAS}

BRASIL. Ministério da Saúde. Secretaria de Atenção à Saúde. Departamento de Atenção Básica. Hipertensão arterial sistêmica para o Sistema Único de Saúde. Brasília: Ministério da Saúde, 2006a. 58p. (Cadernos de Atenção Básica; 16) (Série A; Normas e Manuais Técnicos).

. Secretaria de Atenção à Saúde. Departamento de Atenção Básica. Diabetes mellitus. Brasília: Ministério da Saúde, 2006b. 56p. (Cadernos de Atenção Básica, n.16) (Série A; Normas e Manuais Técnicos).

. Secretaria de Atenção à Saúde. Manual Técnico do Cadastro Nacional de Estabelecimentos de Saúde (CNES) - Anexo. Brasília, Brasil. 2008. Disponível em: http://sitesms.curitiba.pr.gov.br/saude/areastematicas/cnes/Tabelas\%20Novas.pdf. Acesso em: 08 out.2009.

Manual de hipertensão arterial e diabetes mellitus. Brasília, Brasil, 2002. Disponível em: bvsms.saude.gov.br/bvs/publicacoes/miolo2002.pdf. Acesso em: 15 abr.2008.

CIPRIANO, Dilênia de Oliveira; LIMA, Heidy Vieira; SOUZA, Ana Aparecida dos Santos; SILVA, Maria Cleide Freire Clementino da; SANTOS, Ana Célia Oliveira dos. Freqüência de indivíduos com intolerância à glicose em jejum em um hospital universitário: comparação de critérios diagnósticos. Jornal Brasileiro de Patologia Médica e Laboratorial. Rio de Janeiro, v.42, n.3, p.419-423, dez. 2006. Disponível em: www.scielo.br/pdf/jbpml/v42n6/a03v42n6.pdf. Acesso em: 25 abr. 2008.

FRANCIONI, Fabiane Ferreira; SILVA, Denise Maria Guerreiro Vieira da. O processo de viver saudável de pessoas com Diabetes Mellitus através de um grupo de convivência. Texto contexto- 
enferm. Florianópolis,v.16, n.1, p.105-111, jan./mar. 2007. Disponível em:

http://www.scielo.br/pdf/tce/v16n1/a13v16n1.pdf. Acesso em: 15 fev.2009.

PMF - PREFEITURA MUNICIPAL DE FLORIANÓPOLIS. Sistema de Informação da Atenção Básica. Florianópolis - SC, Brasil. Disponível em:

http://www.pmf.sc.gov.br/saude/index2.php?secretaria=siab. Acesso em: 19 out.2008.

SILVA, Denise Maria Guerreiro Vieira da. Narrativa de viver com diabetes mellitus: experiências pessoais e culturais. Tese de Doutorado. Florianópolis, UFSC/Programa de Pós-graduação em Enfermagem, 2001.

SILVA, Denise M. G. Vieira; MEIRELLES, Betina H. S. Atenção a saúde de pessoas com diabetes mellitus e hipertensão arterial na unidade local de saúde da Lagoa da

Conceição. Projeto de extensão: Departamento de Enfermagem - UFSC: Florianópolis. 2008. 